\title{
Preliminary analysis on an IVHM approach for prognosis of high-pressure filters for hydraulic Power Control Modules of helicopters
}

\author{
Andrea De Martin ${ }^{1, *}$, Giovanni Jacazio ${ }^{l}$, Massimo Sorli ${ }^{l}$ and Vincenzo Surdo \\ ${ }^{1}$ Politecnico di Torino, Department of M echanical and A erospace Engineering, Corso Duca degli \\ A bruzzi 24 , Torino, I taly
}

\begin{abstract}
The process of filtration is critical to ensure long operative life to on-board hydraulic equipment, especially in rotating-wing application where the severe vibratory environment lead to accelerated wear of the mechanical components and hence an increased production of debris. Filtration is obtained by mechanically separating the physical contaminants from the hydraulic fluid by means of filters, which hence tend to clog under prolonged usage. Filter replacement has been so far pursued through scheduled maintenance strategy, which however have proven to be rather cost-ineffective. To transition to a Condition-Based $M$ aintenance, new Prognostics and $\mathrm{H}$ ealth $\mathrm{M}$ onitoring frameworks need to be developed. The paper deals with the feasibility analysis of such a system based on a highfidelity simulation environment, rigorous description of the operating conditions and state-of-the-art algorithms.
\end{abstract}

\section{Introduction}

The vast majority of the in-service aircrafts makes use of a centralized architecture to provide the hydraulic power needed to operate the actuators employed to regulate the behaviour of the flight control system, to retract and steer the landing gears and to provide additional functions. Although some applications of Electro-Hydrostatic Actuators have recently emerged for fixed-wing vehicles, their application is still limited to back-up usage and do not avoid the need of a central hydraulic power unit; the same can be said for helicopters, where centralized architectures are still the safest and almost universally adopted solution to supply flight-control actuators. The centralized architecture is built upon the Hydraulic Power Control Modules (PCM)s, complex devices integrating the functions of several components to provide the intended flow-rate to the users, condition the fluid through filters and eventually heat-exchangers while ensuring a safe pressure level within the vehicle by means of properly placed relief valves and accumulators. One of the most critical function of the power unit is in fact to properly filter the impurities and the debris present in the fluid to achieve the long-term functionality of the supplied systems under prolonged usage. Unchecked amount of contaminants can be reason of severe danger to the integrity of some of the most delicate components of the actuation systems, such as the servo valves; free-roaming debris can damage the actuators seals, and alter the geometry of control components by scratching surfaces and edges of the smallest hydraulic 
channels (i.e. the metering edge of a servovalve). This is especially true for rotating-wings applications, where the severe vibratory environment tends to increase the wear rate of the component, and hence raising the production of metallic and non-metallic debris. Filtration allows to separate the solid, dangerous particles from the hydraulic fluid by mechanically segregating the debris by means of grids, metallic meshes or porous materials. The segregated material is held inside the filter, which hence tends to clog under prolonged usage. So far, filter substitution follows a scheduled maintenance scheme, aimed at avoiding the possibility of clogging during flight. Practical experience however suggests that this approach tends to be excessively conservative, causing additional costs and unnecessary vehicle downtimes due to the replacement of well-operating filters. The development of novel prognostic solutions to optimize the filter maintenance operations would hence provide advantages in terms of cost reduction and system availability. Literature on the subject is so far almost non-existent and limited to experimental studies in laboratory conditions [1,2]. The study of novel PHM techniques for PCMs is further complicated by the lack of localized sensors, which makes unavailable the direct measurement of physical quantities instrumental to the health monitoring analysis. We hence propose an Integrated Vehicle Health Monitoring approach, where signals coming from the actuators are used in conjunction with the information available within the power unit to obtain better estimate of the filter clogging status. The paper deals with a preliminary study on the subject aimed at assessing the feasibility of such PHM system and highlight the most critical points to be addressed in further stages of the research, using an approach mutated from previous experience on electro-hydraulic actuators [3]. To meet these goals a dynamic model of the system is first presented and discussed; particular care is reserved to obtain an accurate representation of the uncertainties associated with the experimental measure and the physical processes. Hence, a feature is selected by state-ofthe-art metrics and used within a data-driven fault detection system. Prognosis is then achieved through particle-filtering techniques and tested against realistic degradation patterns. Results are then discussed and further development proposed.

\section{System Architecture}

The architecture of the PCM under analysis refers to a real case which represent on of the most common configurations for fixed wing application; its functional scheme is reported in Fig.1. A high-pressure, variable displacement pump is used to convey the oil from the pressurized tank reservoir to the user; the fluid must pass through a check-valve used to avoid backflow issues and is forced through the high-pressure filter (1a).

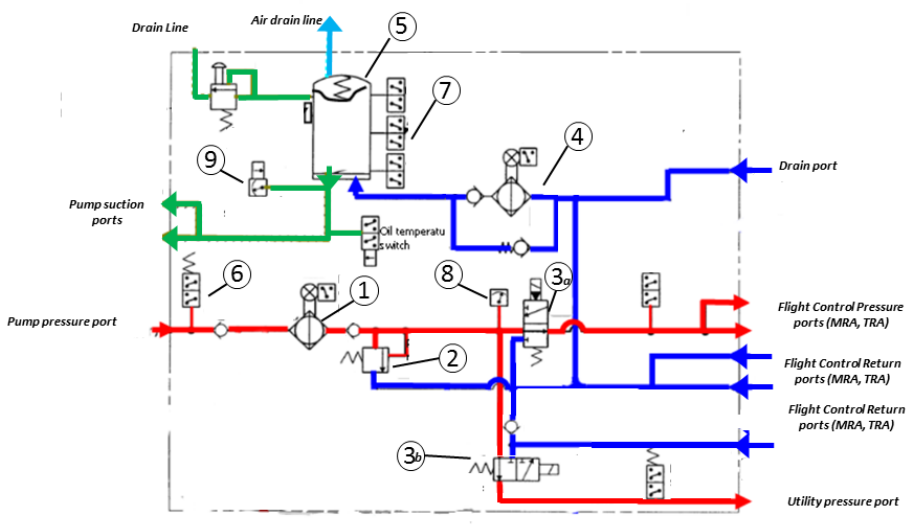

Fig.1. Power Control M odule architecture. 
A pressure relief valve (2) is inserted in derivation to avoid overpressures and a circuit shutoff valve (3a) is used to eventually disconnect the flight control users (Main Rotor Actuators MRA and Tail Rotor Actuators TRA) from the PCM supply line. A utility shutoff valve (3b) provides the same functions to the hydraulic line connected with the accessories. A low-pressure filter (4) is put on the return line, which brings back the oil to the reservoir (5). The system comes equipped with a set number of sensors, most of which provides only a digital on/off output; to this category belong the pressure switches (6) positioned on the supply line to provide alert in case of failure conditions, and the oil level indicators mounted on the reservoir (7). The system is then equipped with a pressure transducer on the supply line (8), differential pressure indicators on the filters and an oil temperature sensor situated in the reservoir (9). Not represented in the scheme, the actuators operating on the main and the tail rotors follows a new configuration based on fly-by-wire hydraulic actuators. These actuators are equipped with their own set of sensors used for monitoring and control. For the application under analysis are of particular interest the Linear Variable Differential Transducers (LVDT) used to measure the actuators positions, which can be used to infer the actuators speed and provide valuable information to the Health Monitoring system.

\section{System Model}

The model of the entire system has been prepared starting from well-known mathematical expressions often used in literature to describe the dynamic behaviour of each of the involved components. Some of the components have been modelled through detailed investigation of their dynamics, while a few of them have been introduced only as functional blocks; justifications for this choice are provided in the rest of the section. The axial pump providing the required flowrate to the whole hydraulic system is a nine-pistons, variable displacement device. Driven directly by the helicopter's engine through its gearbox it is used to transform part of the mechanical power produced by the propulsion system into hydraulic power then used by the several actuators present on board. The axial pistons solution with variable displacement is currently the most adopted in PCM form on-board duty, since it provides consistent performances in high-pressure environments and allows to obtain higher efficiency by limiting the supplied flow-rate to the strictly necessary, avoiding the need of an always metering pressure relief valve. By solving the mechanical equilibrium of the swashplate and assuming laminar leakages between delivery and inlet, the instantaneous flow-rate supplied by the pump can be re-written as a function of the tilt angle of the swashplate $\vartheta$, of the volume of the $i$-th chamber $V_{i}$, of the pump shaft rotation $\alpha$ and of the pressure drop across the pump $\Delta P_{p}$. The hydraulic lines have been modelled as combinations of hydraulic resistances and hydraulic capacities. Due to the length of the pipes linking the PCM with the users, distributed losses are not negligible and computed through the Darcy Law, while localized pressure drops due to turns and other geometry variations are computed as detailed in [4]. With regards to valves, check-valves and the pressure relief valve have been physically modelled, while shut-off valves have been simply described as digital gains activating or deactivating the users. The behaviour of check-valves and the pressure relief valve is determined through the description of both the mechanical dynamics and of the flow characteristics of the valve, described as a function of the local Reynolds' number [4]. The discharged flow-rate also depends on the fluid density, mostly function of the oil temperature. The valve lift can hence be computed and is limited to a maximum value defined by the device geometry; its behavior depends on the mechanical equilibrium defined by the force generated by the pressure drop across the metering edge $\Delta P_{v}$ and the section $A_{v}$, the poppet mass $m_{v}$, the viscous friction $c_{v} \dot{x}$ and the 
effect of the spring stiffness $k_{v}$ and pre-load $F^{*}$, whenever a spring is present. The reservoir has been physically modelled as well considering it pressurized as a spring-based accumulator. Both MRA and TRA flight control systems have been modelled in-depth through a high-fidelity description similar to that reported in [5] for similar components. However, to keep the computational effort and time in check, these models have been used only to tune the parameters of the equivalent hydraulic resistance representing the actuators behavior. The passage area of the localized hydraulic resistance is continuously regulated to ensure that the required flow-rate passing through is equivalent to that needed to move each actuator at the desired speed. The desired speed signals of each actuators are provided in input to the simulations and are obtained through the analysis of the expected commands spectrum. Additional resistances are used to describe the flow lost due to leakages, describing both the laminar behavior of the fluid through the Hagen-Poiseuille law, while the turbulent one is described as a function of the Reynold's number [6,7].

\section{Filtration model and occlusion process}

Lubricating fluids get inevitably contaminated during long-sustained operations; metallic and non-metallic debris are generated by wear of mating surfaces in relative motion, external particles could enter the system regularly (as in the case of lubricating systems for aeronautic engines) or in case of faulted sealing conditions (as for flight control systems). In time, these particles can negatively affect the chemical and mechanical properties of the lubricant itself [7], cause rapidly accelerating wear of sealing components and critically damage or occlude the most delicate or narrow hydraulic components. Filters have the task to physically separate these impurities from the hydraulic fluid; once separated they are not expelled, and hence naturally occlude the filter in time. Eventually, situation becomes such that the filer must be replaced to allow the operability of the hydraulic lines. The filters of the investigated system belong to the multi-layered type, which is obtained by combining the effects of several filtering grids. Filters have been modelled as a combination of hydraulic resistances, which scheme is depicted in Fig.2. The total pressure drop across the filter $\Delta P_{f}$ can be thought as the sum of the pressure drop across the inlet and outlet interfaces $\Delta P_{f, \text { in }}$ and $\Delta P_{f, o u t}$, and the pressure drop across each layer due to localized $\Delta P_{f, L}$ and distributed losses $\Delta P_{f, D}$. The inlet and outlet pressures drop are due to the geometry variations that the fluid sees when entering and leaving the filter case. These resistances have been modelled as localized losses characterized by a discharge coefficient function of the Reynolds' number. The localized pressure drop due to the geometry variations introduced by each filter layer is described as suggested in [4], while the distributed pressure drop is modelled according to the Hagen-Poiseuille Law under the assumption of laminar flow within the filter's cells. Model parameters have been tuned to recreate the characteristics of a real filter, as depicted in Fig.2. Filters occlusion occurs naturally due to the accumulation of debris and other contaminants. To describe the variation of the filter's properties due to on-going contamination we can refer to the models provided by Ergun [8] and Carman [9]. The parameters required to apply these models are extremely difficult to predict a-priori, since they are associated with the precise simulation of the wear processes in several hydraulic and mechanical components, which is not currently possible to pursue for the lengthy time-span that must be investigated in PHM analysis. As such, we introduced in our model the defect as a reduction of the filter's area $F_{1}$, while the amount of debris generated by the flight control system was estimated by applying the Archard's Law to the actuators. Parameters for the Archard's law, such as the actuators' speed and pressures are known because are input to the model. 


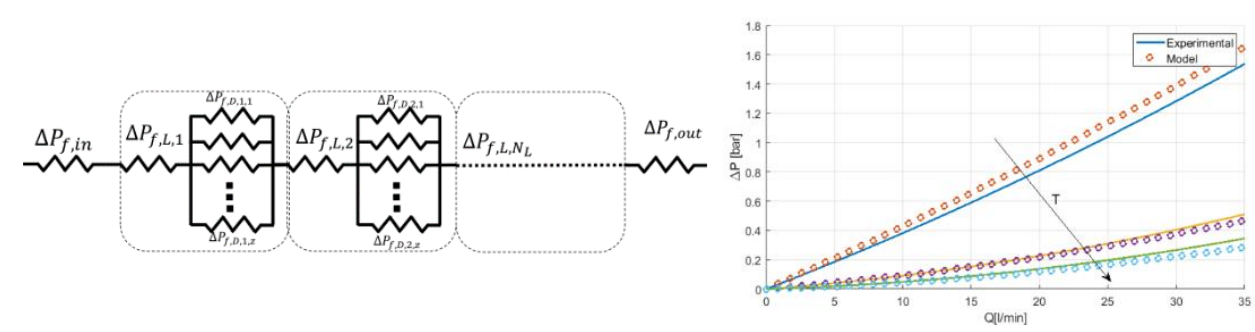

Fig.2. Filter model and comparison between simulated and experimental characteristics

\section{Operational scenarios and uncertainty sources}

The model described in Sections 3 and 4 has been used to build the database on which the PHM system is designed. In building the database, it is mandatory to properly assess and address the uncertainty sources acting on the available signals, since PHM systems must inevitably deal with stochastic processes and non-deterministic reasoning. As such, simulations have been performed by over imposing noise of variable type and entity on all the physical and geometrical parameters within the system, as well as on all the expressions defining the variation of one or more physical properties in dependence of another variable (i.e. the dependence of the fluid viscosity on temperature). Moreover, the operational scenario was perturbated by over imposing a Gaussian noise with zero mean and variance equal to the $10 \%$ of the nominal values each operational parameter (temperature, flow-rate requirements...). To properly perform the preliminary design of a PHM system and to pursue the feasibility analysis, two distinct data-bases have been prepared. The first database is used characterize the system behaviour and select the proper features to be used in PHM; we will refer to this data-base as the "feature selection" one. The second data-base is used to test the prognostics and diagnostics routines chosen for this application, and is addressed as the "testing" one. The "feature selection" database is built by artificially increasing the high-pressure filter occlusion level by a fixed state up to reaching the complete jamming of the hydraulic line. For each degradation level, 200 simulations have been performed according to a realistic operational scenario; each of the 200 simulations belongs to one of the possible operational conditions, and the number of simulations for each condition is proportional to the expected percentage of the operating time they are expected to employ. In this way, the "feature selection" database is representative of the system behaviour and of its expected probability distribution for each occlusion level investigated. The "testing" database was instead obtained by adding the time variable to the simulation and leaving the occlusion of the filters free to evolve according to amount of debris produced by the model. The operational scenarios have been randomly mixed, but their occurrence remains proportional to the operating time percentage reported before.

\section{IVHM strategy for PHM}

To characterize the high-pressure filter behaviour during any operational conditions of the PCM there is the need to assess which signals are needed and which might be desirable, and between those which are available and which are not. As well described by the equations provided in section 5, the pressure drop across the filter is heavily dependent on three major factors; the passing flow-rate, the occlusion level and the fluid viscosity, itself dependent on the oil temperature. Of this information, only the pressure drop can be obtained locally by already existent sensors, while the temperature measure is obtainable through the sensor mounted within the reservoir. Unfortunately, no information is directly available on the 
flow-rate. We propose two possible solutions to this issue. The first and most practical solution for newly designed systems is to insert a calibrated orifice downstream the orifice along a differential pressure sensor, hence realizing a virtual sensor for the flow-rate measure. This solution would have the benefit of being close to the component to monitor, but comes with additional costs and decreased reliability. Moreover, it is not suitable for legacy equipment or in-service systems. Another possibility is represented by an IVHM approach to the problem. While it is true that no flow-meter are equipped on flight control actuators or PCMs, we can infer the flow-rate passing through the filter by using the signals coming from the supplied systems. In particular, the flow-rate entering in the actuation system is strictly correlated with the actuators speed minus the amount of fluid lost due to leakages. Luckily, the position of the flight control actuators can be either directly measured or inferred. Hence, actuators speed and hence, the net flow-rate they are using, can be derived by differentiating this signal in time. Both approaches are investigated and the results compared in the following paragraph. Signals obtained in the "feature selection" database have been used to search for a feature able to correctly represent the degradation behaviour during its progression. According to [10], a suitable feature must be highly sensitive to the fault inception, highly correlated with the fault evolution into a failure and present high signal-to-noise ratio. Conversely, it should present low correlation with the behaviour of other faults affecting the system. Between a high number of possible candidates, we focused our attention on two features, which expressions are reported in equations (1) and (2).

$$
\begin{aligned}
& F_{c o}=K_{\text {feat }}-\frac{\mu_{t h} Q_{c o}}{\Delta P_{f}} \\
& F_{A S}=K_{\text {feat }}-\frac{\mu_{t h} \sum_{i=1}^{Z} v_{i} A_{i}}{\Delta P_{f}}
\end{aligned}
$$

In both cases, $\mu_{t h}$ is the theoretical fluid viscosity provided its temperature, while $K_{\text {feat }}$ is a constant equal to the maximum expected value of the negative term in the respective equations. The theoretical fluid viscosity can differ from the value used in the simulations due to noise over the experimental characteristics and possible air presence. $Q_{c o}$ is the flowrate estimate computed through the virtual sensor eventually placed downstream the filter, while $v_{i}, A_{i}$ and $Z$ are respectively the inferred actuators speed, cylinders areas and total number. Features performances have been evaluated also by studying their behaviour during fault detection. Fault detection is the process through which we aim at detecting the early insurgence of a fault, and is the first step of the diagnostic process, usually completed by the fault isolation and identification processes. Dealing with only one fault type, the last steps are not pursued. The employed fault detection routine is a purely data-driven algorithm, which exploits the knowledge of the system behaviour in healthy conditions to build a baseline for the considered features, hence comparing this baseline with a running distribution of said features. Faulty filter conditions are declared once the $90 \%$ of the running distribution overcomes the $90^{\text {th }}$ percentile of the healthy baseline. An example of the output provided by the routine is depicted in Fig.3, where the results of both proposed features are reported. The baseline distributions (in white), the running distributions at the fault detection instant (in red) and those at time $t=875 \mathrm{~h}$ (in dark grey) are reported in the two window at the top, while in the bottom window we may find the behaviour of the confidence level associated with the fault declarations, the alarm flag consequently arisen and the expected degradation growth (where 0 stands for no degradation and 1 for complete occlusion of the filter). Both features are able to detect the filter occlusion when it is still under the $40 \%$ of its critical value; the feature derived from the addition of a virtual flow- 
rate sensor is as expected more efficient and allows for earlier observation of the degradation.

\section{Prognosis}

Prognosis deals with the estimate of the Remaining Useful Life of the faulted component; since any prediction is inherently uncertain, the more rigorous result of prognosis is the expected probability distribution of the time-of-failure; as such, the prognostic algorithm must be able to deal with the data uncertainty provided by the process and measure noise, and must be able to characterize this uncertainty and forecast its behaviour in time. The proposed prognostic framework is based on a nonlinear process (fault / degradation) model, and a Bayesian estimation method using particle filtering and real-time measurements [11], based on the Sequential Importance Resampling (SIR) scheme. Although more advanced versions of this algorithm are available in literature [12], the preliminary nature of this study lead us to use the less-computing demanding strategy for particles resampling. Long-term predictions are used to estimate the probability of failure in a system given a hazard zone that is defined via a probability density function with lower and upper bounds for the domain of the random variable. An in-depth mathematical description of the process can be found in [13] and [14]. The algorithm makes use of degradation models that are continuously tuned to compute the current a-priori state of the system and to perform the iterative calculation that leads to the long-term prediction. Auto-tuned models are required to describe and follow changes in the degradation process and to describe the process and measurement noise. An example of its output, obtained for the feature $F_{c o}$, is depicted in Fig.3, where the behaviour of the estimated degradation values, the related feature distribution and their projections are represented along the RUL distribution and associated risk evaluation. Both features fares rather well according to traditional metrics provided by [15], with the feature obtained through the additional sensor providing an average Relative Accuracy (RA) of the prediction equal to $78.2 \%$ and the one based on the IHVM approach sporting an RA of $75.1 \%$. The lower performances of the IVHM features were expected and due to the additional noise on the signals provided by the user leakage and by a lower accurate estimate of the supplied flow-rate.
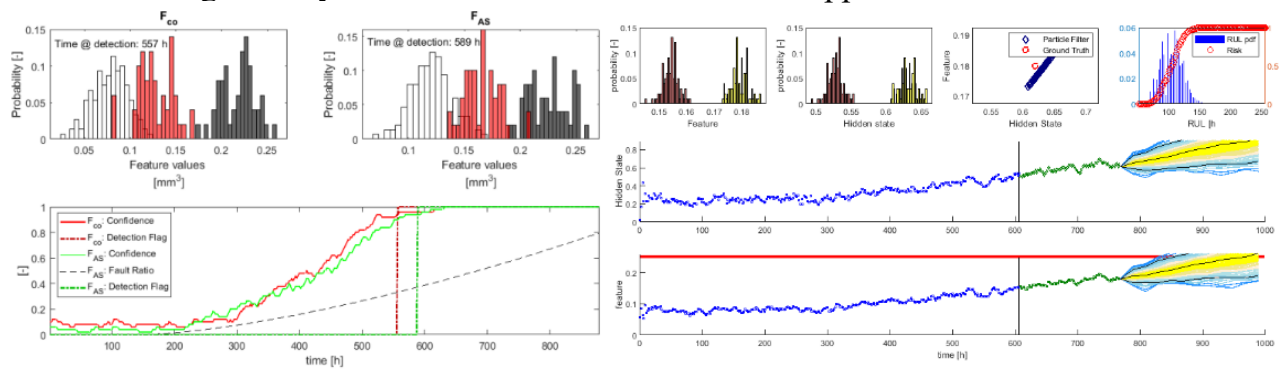

Fig.3. Results of F ault $D$ etection and Failure prognosis routines

\section{Conclusions}

The study provided positive results regarding the feasibility of a PHM system to monitor and forecast the evolution of the high-pressure filter occlusion in PCM for helicopter applications and helped the identification of a rigorous methodology for preliminary studies on prognostics issues. Two solutions were investigated, with the IVHM one providing competitive results with respect to the addition of sensors. Further development is however required to realize a first technological demonstrator; a more 
complete simulation environment must be built and run with command sequences coming from simulated or real mission profiles. Additional efforts need to be reserved to the algorithms as well, to verify their behaviour in case of failures or faults in one or more of the PCM users and to better characterize and validate the obtained RUL distribution estimates.

\section{References}

[1] Z. Skaf, O.F. Eker, I.K. Jennions, A Simple State-Based Prognostic Model for Filter Clogging, in: Procedia CIRP, 2015.

https://doi.org/10.1016/j.procir.2015.08.094.

[2] O.F. Eker, F. Camci, I.K. Jennions, Physics-based prognostic modelling of filter clogging phenomena, Mech. Syst. Signal Process. (2016).

https://doi.org/10.1016/j.ymssp.2015.12.011.

[3] S. Autin, J. Socheleau, A. Dellacasa, A. De Martin, G. Jacazio, G. Vachtsevanos, Feasibility Study of a PHM System for Electro-hydraulic Servo- actuators for Primary Flight Controls, in: Annu. Conf. Progn. Heal. Manag. Soc., 2018: pp. 119.

[4] I.E. Idelchik, E. Fried, Handbook of hydraulic resistance: Second edition, 1986.

[5] A. De Martin, A. Dellacasa, G. Jacazio, M. Sorli, High-Fidelity Model of ElectroHydraulic Actuators for Primary Flight Control Systems, in: Proc. 2018

Bath/ASME Symp. Fluid Power Motion Control FPMC2018 Sept. 12-14, 2018, Univ. Bath, United Kingdom, 2018: p. V001T01A058.

https://doi.org/10.1115/fpmc2018-8917.

[6] M. Jelali, A. Kroll, Hydraulic Servo-systems, 2003. https://doi.org/10.1007/978-14471-0099-7.

[7] G. Jacazio, A. De Martin, Influence of rotor profile geometry on the performance of an original low-pressure gerotor pump, Mech. Mach. Theory. 100 (2016) 296-312. https://doi.org/10.1016/j.mechmachtheory.2016.02.012.

[8] S. Ergun, Fluid flow through packed columns, Chem. Eng. Prog. (1952).

[9] P.C. Carman, Fluid flow through granular beds, Process Saf. Environ. Prot. Trans. Inst. Chem. Eng. Part B. (1997).

[10] G. Vachtsevanos, F. Lewis, M. Roemer, A. Hess, B. Wu, Intelligent Fault Diagnosis and Prognosis for Engineering Systems, 2007.

https://doi.org/10.1002/9780470117842.

[11] M.E. Orchard, G.J. Vachtsevanos, A particle-filtering approach for on-line fault diagnosis and failure prognosis, Trans. Inst. Meas. Control. (2009). https://doi.org/10.1177/0142331208092026.

[12] D.E. Acuña, M.E. Orchard, Particle-filtering-based failure prognosis via sigmapoints : Application to Lithium-Ion battery State-of-Charge monitoring, Mech. Syst. Signal Process. 85 (2017) 827-848.

https://doi.org/10.1016/j.ymssp.2016.08.029.

[13] D.E. Acuña, M.E. Orchard, A theoretically rigorous approach to failure prognosis, in: Proc. 10th Annu. Conf. Progn. Heal. Manag. Soc. 2018, 2018.

[14] A. De Martin, G. Jacazio, M. Sorli, Enhanced Particle Filter framework for improved prognosis of Electro-Mechanical flight controls Actuators, in: 3rd Eur. Conf. Progn. Heal. Manag. Soc., 2017: pp. 1-10.

[15] A. Saxena, J. Celaya, E. Balaban, K. Goebel, B. Saha, S. Saha, M. Schwabacher, Metrics for evaluating performance of prognostic techniques, in: 2008 Int. Conf. Progn. Heal. Manag. PHM 2008, 2008. https://doi.org/10.1109/PHM.2008.4711436. 